\title{
AREA OF CURVED SURFACES
}

\author{
BY

\section{JAMES PIERPONT}

\section{§1. Introduction.}

Let the surface $S$ be defined by the equations

$$
x=f(u, v), \quad y=g(u, v), \quad z=h(u, v),
$$

where $f, g, h$ are continuous single valued functions in a complete limited region $\mathfrak{A}$. In case their first derivatives are continuous in $\mathfrak{A}$, i. e., in case $S$ has a continuously turning tangent plane, the area $\dagger$ of $S$, which we may without ambiguity denote by the same letter, is

$$
S=\int_{\Re} 1 \overline{A^{2}+B^{2}+C^{2}} d u d v
$$

where

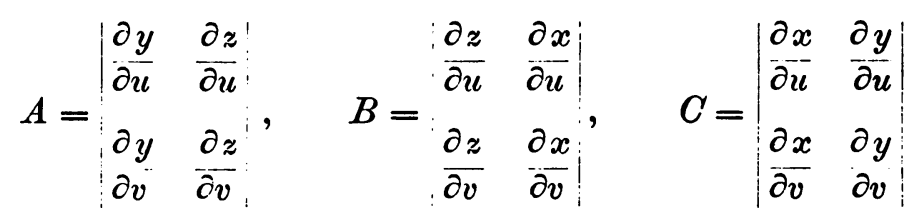

The first attempt to show that the formula (1) still holds in case the derivatives of the coördinates are not continuous was by STOLz. $\ddagger$ He showed the validity of $(1)$ in the case that the six partial derivatives $\partial x / \partial u, \partial x / \partial v$, etc., are limited in $\mathfrak{A}$, and are continuous except at a finite number of points, or on a finite number of ordinary curves.

In the present paper I wish to show that the formula (1) holds for inuch wider cases.

Before doing this, it is proper to call attention to the fact that the definition of the area of a curved surface varies greatly with different authors. For example, no two of the four authors cited in the second footnote employ the same

* Presented to the Society A pril 28, 1906 . Received for publication June 2, 1906.

$\dagger$ Hölder, Beitrüge zur Potentinltheorie, Dissertation, Stuttgart, 1882, p. 29, seq. ; JoRnds, Cours d'Analyse, Paris, 1893, vol. 1, p. 146, seq. ; STolz, Grundzïge, Leipzig, 1899, vol. 3, p. 239, seq. ; Gounsat, Cours d'Analyse, Paris, 1902, vol. 1, p. 306, seq. ; or English trauslation by Henrick, Ginn and Co., p. 272 ; Vallee Poussin, Cours, vol. 2.

$\ddagger$ These Transactions, rol. 3 (1902), p. 34, seq. 
definition, although the four definitions lead to the same result in the case that the surface has a continuously turning tangent plane.

In seeking to remove the restriction of continuity as far as possible, I have found myself forced to adopt a type of definition similar to those employed by HöLdER and STOLz, which may be described briefly as follows:

Let us effect a rectangular division * of the $u, v$ plane of norm $d$. By drawing the diagonals of these rectangles, we divide the plane into little right angled triangles $t_{\kappa}$. Such a division we shall call a quasi rectangular division of norm $d$. Corresponding to a $t_{\kappa}$ whose vertices $P_{0}, P_{1}, P_{2}$ lie in $\mathfrak{A}$, is a plane triangle $\tau_{\kappa}$ inscribed in the surface $S$, and thus to a quasi rectangular division $D$ of $\mathfrak{A}$ corresponds the inscription of a polyhedron in $S$ whose faces are the little triangles, $\tau_{\kappa}$. If $S_{D}$ denotes the area of this polyhedron, the area of $S$ is defined by STOLz to be

$$
\lim _{d=0} S_{D}
$$

The objection to this definition lies in the restriction of the polyhedra. One cannot help asking: would the limit of $S_{D}$ be the same for a system of divisions which are not quasi rectangular? Let us therefore take a more general system of divisions. Let us divide the $u, v$ plane into little triangles in any way, such however that no square of finite size contains an infinite number of triangles. If each triangle can be enclosed in a square of side $d$, we shall call such a division a triangular division of norm $d$. Corresponding to such a division of the $u, v$ plane is a polyhedron inscribed in the surface $S$, whose faces are also triangular, as before. If $S_{D}$ again denote the area of this polyhedron we may investigate the limit (2), and if it exists it may be called the area of $S$.

But when we take the divisions $D$ in this very general manner, this limit does not exist, as Schwarz first pointed out. $\dagger$ Some restriction of $D$ is thus necessary. HöLDER, in the paper already referred to, showed that the limit (2) exists for all divisions $D$ such that no angle of any triangle of $D$ is greater than $\pi-\epsilon$, where $\epsilon(\epsilon>0)$ is taken small at pleasure, and then fixed. As the sines of these angles do not sink below some positive constant, I shall call such triangular divisions positive.

In the following investigation I have not always been able to adopt as general a definition as does Hölder, who considers only the case that the first derivatives of the coördinates are continuous. On the other hand I have striven, whenever possible, to employ triangular divisions much more general than the quasi rectangular divisions of SToLz.

* Cf. the author's Lectures on the Theory of Functions of Real Variables, vol. 1, Ginn and Co., $1905, Z 253$; or the author's papers on Multiple Integrals, these Transactions, vols. $C, 7$ (1905, 1906).

† ScHWARz, Werke, vol. 2, pp. 309 and 369. 


\section{§2. Regular surfuces.}

Let us begin by considering a class of surfaces having at each point a tangent plane, which however does not necessarily turn continuously. Such surfaces may be called regular.

Let the coördinates $x, y, z$ be one valued totally differentiable * functions in I. Let

$$
P_{0}=\left(u_{0}, v_{0}\right), \quad P_{1}=\left(u_{0}+\delta^{\prime}, v_{0}+\eta^{\prime}\right), \quad P_{2}=\left(u_{0}+\delta^{\prime \prime}, v_{0}+\eta^{\prime \prime}\right)
$$

be the vertices of $t_{\kappa}$. The area of the corresponding triangle, $\tau_{\kappa}$, is given by

where

$$
\tau_{\kappa}^{2}=X_{\kappa}^{2}+Y_{\kappa}^{2}+Z_{\kappa}^{2}
$$

$$
2 X_{\kappa}=\left|\begin{array}{lll}
y_{0} & z_{0} & 1 \\
y_{1} & z_{1} & 1 \\
y_{2} & z_{2} & 1
\end{array}\right|=\left|\begin{array}{ll}
y_{1}-y_{0} & z_{1}-z_{0} \\
y_{2}-y_{0} & z_{2}-z_{0}
\end{array}\right|=\mid \begin{array}{cc}
\Delta^{\prime} y & \Delta^{\prime} z \\
\Delta^{\prime \prime} y & \Delta^{\prime \prime} z
\end{array},
$$

while similar expressions hold for $Y_{\kappa}, Z_{\kappa}$. Thus

But

$$
S_{D}=\sum \sqrt{X_{\kappa}^{2}+Y_{\kappa}^{2}+\bar{Z}_{\kappa}^{2}} .
$$

$$
\begin{aligned}
& \Delta^{\prime} x=x_{1}-x_{0}=\frac{\partial x}{\partial u} \delta^{\prime}+\frac{\partial x}{\partial v} \eta^{\prime}+\alpha_{x}^{\prime} \delta^{\prime}+\beta_{x}^{\prime} \eta^{\prime}, \\
& \Delta^{\prime \prime} x=x_{2}-x_{0}=\frac{\partial x}{\partial u} \delta^{\prime \prime}+\frac{\partial x}{\partial v} \eta^{\prime \prime}+\alpha_{x}^{\prime \prime} \delta^{\prime \prime}+\beta_{x}^{\prime \prime} \eta^{\prime \prime},
\end{aligned}
$$

while similar expressions hold for the other increments. Thus

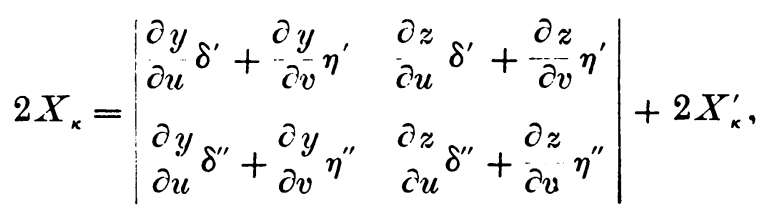

where $X_{\alpha}^{\prime}$ denotes a sum of several determinants involving the infinitesimals $\alpha_{y}^{\prime}, \alpha_{y}^{\prime \prime}, \beta_{z}^{\prime}, \beta_{z}^{\prime}$. Similar expressions hold for $Y_{\kappa}, Z_{\kappa}$. We get thus

$$
X_{\kappa}=A_{\kappa} t_{\kappa}+X_{\kappa}^{\prime}, \quad Y_{\kappa}=B_{\kappa} t_{\kappa}+Y_{\kappa}^{\prime}, \quad Z_{\kappa}=C_{\kappa} t+Z_{\kappa}^{\prime},
$$

where $A_{\kappa}, B_{\kappa}, C_{\kappa}$ denote the values of the determinants $A, B, C$ at the point $P_{0}$. Thus

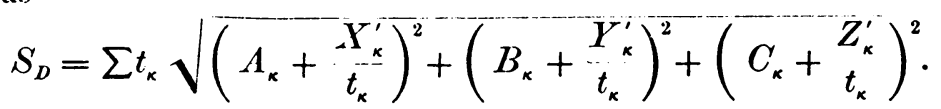

* Lerturis, § 423. 
Let us suppose that $A^{2}+B^{2}+C^{2}$ remains greater than some positive number, while

$$
\frac{X_{\kappa}^{\prime}}{t_{\kappa}}, \quad \frac{Y_{\kappa}^{\prime}}{t_{\kappa}}, \quad \frac{Z_{\kappa}^{\prime}}{t_{\kappa}}
$$

are in general uniformly evanescent in $\mathfrak{A}$; i. e., let them remain numerically less than some positive number arbitrarily small, for any division $D$ of norm $d \leqq d_{0}$, except in the vicinity of a.discrete set of points in $\mathfrak{A}$. Then

where, in general,

$$
S_{D}=\sum t_{\kappa} \sqrt{A_{\kappa}^{2}+B_{\kappa}^{2}+C_{\kappa}^{2}}+\sum \epsilon_{\kappa} t_{\kappa},
$$

$$
\left|\epsilon_{\kappa}\right|<\epsilon / \overline{\mathfrak{A}} \text {.* }
$$

If now $A, B, C$ are limited and integrable in $\mathfrak{A}$, we have

$$
\lim S_{b}=\int_{\mathfrak{x}} \sqrt{A^{2}+B^{2}+C^{2}} d u d v .
$$

We ask now under what conditions are the expressions (3) in general uniformly evanescent? The answer is pretty evident from the example given by Schwarz. In fact the equation of the tangent plane at the point $x_{0} y_{0} z_{0}$ is

$$
A_{\kappa}\left(x-x_{0}\right)+B_{\kappa}\left(y-y_{0}\right)+C_{\kappa}\left(z-z_{0}\right)=0 .
$$

On the other hand, the plane of the triangle $\tau_{k}$ is

or

$$
\left|\begin{array}{llll}
x & y & z & 1 \\
x_{0} & y_{0} & z_{0} & 1 \\
x_{1} & y_{1} & z_{1} & 1 \\
x_{2} & y_{2} & z_{2} & 1
\end{array}\right|=0,
$$

or finally

$$
x X_{\kappa}+y Y_{\kappa}+z Z_{\kappa}+U_{\kappa}=0 \text {, }
$$

$$
x\left(A_{\kappa}+\frac{X_{\kappa}^{\prime}}{t_{\kappa}}\right)+y\left(B_{\kappa}+\frac{Y_{\kappa}^{\prime}}{t_{\kappa}}\right)+z\left(C_{\kappa}+\frac{Z_{\kappa}^{\prime}}{t_{\kappa}}\right)+\frac{U_{\kappa}}{t_{\kappa}}=0 .
$$

Thus for (3) to converge in general uniformly to zero, it is necessary and suffcient that the planes of the faces of the inscribed polyhedra converge in general uniformly to tangent planes.

Let us call a triangular division such that the faces of the corresponding polyhedra converge in general uniformly to tangent planes a regular division. We have now the following:

Theorem 1. Let the coördinates $x, y, z$ be one valued totally differentiable functions of the parameters $u, v$ in the limited complete region $\mathfrak{A}$, such that

* $\overline{\mathfrak{A}}=\overline{\mathrm{Con} t} \mathfrak{A}$ denotes upper content of $\mathfrak{A}$. Cf. Lestures, $\& 702$. 
$A^{2}+B^{2}+C^{2}$ is greater than some positive constant, and is limited and integrable in $\mathfrak{A}$. Then

$$
S=\lim _{d=0} S_{D}=\int_{\mathfrak{a}} \sqrt{A^{2}+B^{2}+C^{2}} d u d v,
$$

$D$ denoting a regular triangular division of norm $d$.

If we call this limit area, we have at once a number of its properties. We mention only the following :

Theorem 2. Let $\mathfrak{A}_{1}, \ldots, \mathfrak{A}_{m}$ be unmixed ${ }^{*}$ partial complete regions of $\mathfrak{A}$, whose union is $\mathfrak{A}$. Let $S_{1}, \ldots, S_{m}$ be the pieces of $S$ corresponding to them. Then each $S_{\kappa}$ has an area, and their sum is $S$.

TheoRem 3. Let $\mathfrak{A}_{\lambda}$ be an unmixed partial complete region of $\mathfrak{A}$ depending on a parameter $\lambda \doteq 0$, such that $\lim \overline{\mathfrak{A}}_{\lambda}=\overline{\mathfrak{A}}$. Then

$$
\lim _{\lambda=0} S_{\lambda}=S
$$

Theorem 4. The area of $S$ remains unaltered when $S$ is subjected to a displacement or when the parameters $u, v$ are subjected to the transformation of theorems 31, 32 in the author's paper $\dagger$ on Improper Integrals.

\section{§3. Irregular surfaces.}

We turn now to certain surfaces which do not have tangent planes at every point, i. e., surfaces for which one or more of the first partial differential coefficients of the coördinates do not exist at certain points, and which may be styled irregular surfaces.

TheоReм 5. Let the coördinates $x, y, z$ be one valued functions, having limited total difference quotients $\ddagger$ in the limited complete region $\mathfrak{A}$. Let $D$ be a positive division of norm $\leqq$ some number $d_{0}$. Then

is finite, and evanescent with $\overline{\mathfrak{A}}$.

$$
\max S_{D}
$$

For, let the difference quotients remain $\leqq \mu$. We have

But

$$
S_{D} \leqq \sum\left|X_{\kappa}\right|+\sum\left|Y_{\kappa}\right|+\sum\left|Z_{\kappa}\right| \text {. }
$$

$$
\begin{aligned}
&\left|X_{\kappa}\right|=\frac{1}{2}\left|\Delta^{\prime} y \Delta^{\prime \prime} z-\Delta^{\prime} z \Delta^{\prime \prime} y\right| \leqq \frac{1}{2}\left\{\left|\Delta^{\prime} y\right| \cdot\left|\Delta^{\prime \prime} z\right|+\left|\Delta^{\prime} z\right| \cdot\left|\Delta^{\prime \prime} y\right|\right\} \\
& \leqq \mu^{2} \overline{P_{0} P_{1}} \cdot \overline{P_{0} P_{2}}=\mu^{2} t_{\kappa}\left|\csc \theta_{\kappa}\right| ;
\end{aligned}
$$

where $\theta_{\kappa}$ is the angle made by the sides $\overline{P_{0} P_{1}}, \overline{P_{0} P_{2}}$. As $D$ is a positive

* Lectures, § 711.

† These Transactions, vol. 7 (1906), p. $17 \%$.

$\ddagger$ Lectures, § 708. 
division, one of the angles of $t_{\kappa}$ is such that $\csc \theta_{\kappa}$ is numerically less than some positive number $M$. Thus

$$
\left|X_{\kappa}\right|<\mu^{2} M t_{\kappa} \text {, }
$$

where $\mu, M$ are independent of $\kappa$ and $d$. Similar relations hold for $\left|Y_{\kappa}\right|$, $\left|Z_{\kappa}\right|$; and the truth of the theorem is now obvious.

TheOREM 6. in the limited complete region $\mathfrak{A}$, let the coördinates $x, y, z$ be as in theorem 1, except that at certain points forming a discrete aggregate $\mathfrak{a}$, the first partial differential coefficients do not exist. Let their total difference quotients be limited in $\mathfrak{A}$. Then

$$
\lim _{d=0} S_{D}=\int_{\mathfrak{x}} \sqrt{A^{2}+B^{2}+C^{2}} d u d v
$$

where $D$ denotes a positive regular division of norm $d$.

Let us first show the limit on the left exists. We may choose an unmixed partial region $\mathfrak{B}$ of $\mathfrak{A}$, containing $\mathfrak{a}$ as inner* points, such that $\mathfrak{\mathfrak { B }}$ is as small as we please. Let $\mathfrak{C}=\mathfrak{A}-\mathfrak{B}$, while $S_{C}$ may denote the area of the surface corresponaing to $\mathfrak{C}$. The triangles $t_{\kappa}$ fall into two groups ; first a group $G_{1}$ containing points of $\mathfrak{B}$, and second a group $G_{2}$, containing only points of $\mathfrak{B}$. Then

$$
S_{D}=\sum \sqrt{X_{\kappa}^{2}+Y_{\kappa}^{2}+Z_{\kappa}^{2}}=\sum_{G_{1}}+\sum_{G_{2}} .
$$

But $\overline{\mathfrak{B}}$ may be chosen so small that the first sum is $<\epsilon / 4$ for any $d<d_{0}$. Moreover, by taking $d_{0}$ still smaller if necessary, we have

Hence

$$
\left|\sum_{G_{2}}-S_{C}\right|<\epsilon / 4 \text {. }
$$

$$
\left|S_{D}-S_{C}\right|<\epsilon / 2, \quad\left(d<d_{0}\right) .
$$

Similarly for any other division $D^{\prime}$ of norm $d^{\prime}$,

$$
\left|S_{D^{\prime}}-S_{C}\right|<\epsilon / 2, \quad\left(d^{\prime}<d_{0}\right),
$$

decreasing $d_{0}$ still farther if necessary. Thus

$$
\left|S_{D^{\prime}}-S_{D}\right|<\epsilon, \quad\left(d, d^{\prime}<d_{0}\right) .
$$

Hence $\lim S_{D}$ exists; call it $S$. Since $S$ exists, we may take $d_{0}$ so small that

$$
\left|S-S_{D}\right|<\epsilon / 2 . \quad\left(d<d_{0}\right) .
$$

* Lectures, \& 704, 4. 
This with (4) gives

that is,

$$
\left|S-S_{c}\right|<\epsilon
$$

$$
S=\lim S_{C}=\lim \int_{\mathfrak{x}}=\int_{\mathfrak{x}} \sqrt{A^{2}+B^{2}+C^{2}} d u d v,
$$

by Lectures, $\S 724$.

The preceding theorem takes care of a large class of irregular surfaces whose total difference quotients are limited, either with the original set of parameters or with a new set. When the total difference quotients are not limited, and it is not practical to transform to a new system, whose total difference quotients are limited, the following theorems may be useful. They rest on a new system of divisions which we define as follows. Let us effect a quadrate division of the $u, v$ plane of norm $d$, and take the triangles $t_{\kappa}$ so that for any triangular division $D$ associated with $d$, no square contains more than $n$ triangles, and no triangle lies in more than $\nu$ squares; $n, \nu$ being arbitrarily large constants independent of $d$. Such a division we call a quasi quadrate division of norm $d$.

We shall also need to introduce a new classification of functions according to their variation in $\mathcal{H}$, or along lines parallel to the $u, v$ axes.

Let $D$ be a quadrate division of the $u, v$ plane, of norm $d \leqq d_{0}$. Let $\omega_{\kappa}=\operatorname{osc} \phi(u, v)$ denote the oscillation of $\phi$ in one of the squares of $D$. The extreme

$$
\max _{D} \sum \omega_{\kappa} d
$$

for all divisions $D$ is called* the variation of $\phi$ in $\mathfrak{A}$. If the variation is not only limited, but evanescent with $\overline{\mathfrak{A}}$, we shall say $\phi$ has limited fluctuations in $\mathfrak{A}$. Let $\mathfrak{a}$ denote the points of $\mathfrak{A}$ lying on a line parallel to the $u$ axis. Let us effect a division $D^{\prime}$ of this line into intervals of length $\leqq d$. Let $\omega_{.}^{\prime}=\operatorname{osc} \phi(u, v)$ in one of these intervals. Then

denntes the variation of $\phi$ in $a$.

$$
\tilde{\omega}_{\mathfrak{u}}=\max _{D^{\prime}} \sum \omega_{\kappa}^{\prime}
$$

Let us consider now all the aggregates $a$ lying on lines parailel to the $u$ axis, and suppose $\overline{\mathfrak{a}} \leqq \sigma$, where $\sigma \doteq 0$. If now for each there exists a constant $G$, such that $\bar{\omega}_{\mathfrak{a}}<\sigma G$; that is, if $\tilde{\omega}_{\mathfrak{a}}$ are uniformly evanescent with $\sigma$, we shall say that $\phi(u, v)$ has limited fuctuation with respest to $u$. Similar remarks apply to $v$.

With the aid of these notions we may prove the following theorems.

Theorem 7. Let the coördinates $x, y, z$ be one valued limited functions in the limited complete region $\mathfrak{A}$. Let $x, y$ have limited total difference quotients,

* Lectures, § 709. 
while z has limited variation in $\mathfrak{A}$. Let $D$ denote a quasi quadrate division of norm $d \leqq d_{0}$. Then

is finite.

$$
\max _{D} S_{D}
$$

For, as before

$$
2\left|X_{\kappa}\right| \leqq\left|\Delta_{y}^{\prime}\right| \cdot\left|\Delta_{z}^{\prime \prime}\right|+\left|\Delta_{y}^{\prime \prime}\right| \cdot\left|\Delta_{z}^{\prime}\right| \cdot
$$

But $\mu$ denoting a sufficiently large constant,

$$
\left|\Delta_{y}^{\prime}\right|,\left|\Delta_{y}^{\prime \prime}\right|<\mu d .
$$

Let $\omega_{\imath}=$ osc $z$ in the square $s_{\imath}$. If the triangle $t_{\kappa}$ lies in the squares $s_{\iota_{1}}, \ldots, s_{\iota_{r}}$,

Hence

$$
\left|\Delta_{z}^{\prime}\right|,\left|\Delta_{z}^{\prime \prime}\right| \leqq \omega_{\iota_{1}}+\cdots+\omega_{\iota_{r}}
$$

$$
\left|\Delta_{z}^{\prime}\right|+\left|\Delta_{z}^{\prime \prime}\right| \leqq 2\left(\omega_{\iota_{1}}+\cdots+\omega_{\iota_{v}}\right) .
$$

Thus, $n, \nu, M, M^{\prime}$ denoting sufficiently large constants

$$
\sum\left|X_{k}\right|<\mu n \sum d\left(\omega_{\iota_{1}}+\cdots+\omega_{\iota_{r}}\right),
$$

the summation extending over all squares $s_{\imath}$ containing points of $\mathfrak{A}$. But $z$ having limited variation

$$
\sum \omega_{\imath} d<M \text {. }
$$

Hence

$$
\sum\left|X_{k}\right|, \Sigma\left|Y_{k}\right|<\mu n \nu M
$$

Finally as in theorem 5,

$$
\sum\left|Z_{\kappa}\right|<M^{\prime}
$$

The theorem is thus established.

TheOREM 8. The coördinates $x, y, z$ being as in the preceding theorem, except that $z$ has limited fluctuation in $\mathfrak{A}$, and $D$ denoting a quasi quadrate division of norm $d \leqq d_{0}$,

is finite, and evanescent with $\overline{\mathfrak{A}}$.

$$
\max _{D} S_{D}
$$

The reasoning is the same as in theorem 8, except that now $M$ and $M^{\prime}$ are evanescent with $\overline{\mathfrak{A}}$.

Theorem 9. Let the coördinates $x, y, z$ have limited total difference quotients in $\mathfrak{A}$, while the variation of $z$ along any line parallel to the $u$ or $v$ axes is $<M$. Let $\mathfrak{A}$ lie in a square of side $s \doteq 0$. Then

$$
\max S_{D}<G s \text {; }
$$


where $G$ is some constant independent of $s$, and $D$ is a quasi rectangular division of norm $\leqq d_{0}$.

For, here

$$
2 \sum\left|X_{\kappa}\right| \leqq \sum\left|\Delta^{\prime} y\right| \cdot\left|\Delta^{\prime \prime} z\right|+\sum\left|\Delta^{\prime \prime} y\right| \cdot\left|\Delta^{\prime} z\right|<M^{\prime} \sum \omega_{u} d_{v}+M^{\prime} \sum \omega_{v} d_{u} ;
$$

where $M^{\prime}$ denotes a sufficiently large constant; $d_{u}, d_{v}$ denote the length of sides of one of the triangles $t_{\kappa}$ parallel respectively to the $u, v$ axes, and $\omega_{u}, \omega_{v}$ the oscillation of $z$ along these sides. Since the variation is $\langle M$ in both directions,

Similarly,

$$
\sum \omega_{u} d_{v}=\sum_{v} d_{v} \sum \omega_{u}<M \sum d_{v}<M s .
$$

$$
\sum \omega_{v} d_{u}<M s
$$

The rest of the proof follows as before.

Theorem 10. The symbols having the same meaning as before, except that $z$ has limited fuctuation with respect to $u, v$,

$$
\underset{D}{\operatorname{Max}} S_{D}<G s^{2}
$$

The demonstration is similar to the foregoing.

Following the line of proof used in establishing theorem 6, and employing the theorems just given, we readily prove the following theorems:

Theorem 11. Let $\mathfrak{A}$ be a limited complete region, containing the discrete aggregate $\mathfrak{a} . \quad$ Let $\mathfrak{b}$ be a partial region of $\mathfrak{A}$ containing the points $\mathfrak{a}$, such that $\mathfrak{B}=\mathfrak{A}-\mathfrak{b}$ is exterior to $\mathfrak{a}$, moreover let $\overline{\mathfrak{b}} \doteq 0$. Let the coördinates $x, y, z$ be one valued totally differentiable functions in $\mathfrak{B}$, such that $A^{2}+B^{2}+C^{2}$ never sinks below a positive constant in any $\mathfrak{B}$, is properly integrable in any $\mathfrak{B}$, and improperly integrable in $\mathfrak{A}$. Let $x, y$ have limited total difference quotients and $z$ limited fluctuation in $\mathfrak{b}$. Then

$$
\lim _{d=0} S_{D}=\int_{\mathfrak{x}} \sqrt{A^{2}+B^{2}+C^{2}} d u d v,
$$

where $D$ is any quasi quadrate division of norm $d$.

Theorem 12. Let the symbols have the same meaning as in the preceding theorem except that: 1) a reduces to a finite aggregate; 2) z has limited variation along any line parallel to the $u, v$ axes; 3) $D$ denotes a regular quasi rectangular division. Then

$$
\lim _{d=0} S_{D}=\int_{x}
$$


TheоReм 13. Let the symbols have the same meaning as in theorem 11 except that: 1) $z$ has limited fluctuation with respect to $u, v$ in $\mathfrak{b}$; 2) $D$ denotes a regular quasi rectangular division. Then

$$
\lim _{\imath=0} S_{D}=\int_{\mathfrak{n}}
$$

Theorem 14. If we call the limits in theorems 11, 12, 13 area, the theorems 2, 3, 4 still hold.

RoCK BOUND, SACHEM'S HeAI,

April, 1906. 